\title{
MARINDINEESCHE VERWANTSCHAPS- BETREKKINGEN
}

DOOR

J. VAN DER KOLK.

Wanneer men de taal van een natuurvolk nog woord voor woord geheel uit den mond van dat volk moet afluisteren, opteekenen en ordenen, - zooals dit voor mij het geval was bij de Marindineezen van Nederlandsch Zuid-Nieuw-Guinee, — dan blijkt het vaak dat er vormen bestaan die men tevergeefs in andere talen zoekt. Dit is zelfs het geval voor de spraakkunstige terminologie, b.v. om de vele modi (wijzen) van het Marindineesch werkwoord aan te duiden. Maar hetzelfde heeft men soms nog in erger mate om gewone nomina te vertalen. Men treft n.l. in sommige groepen van substantiva (als b.v. schelpen, visschen etc.) een rijkdom van termen en namen, die eigenlijk tegelijk een armoede aan abstractie en generaliseering beteekent. De natuurmensch benoemt individuen of groepen, en mist vaak de benamingen der «soorten», zoodat vertalen haast onmogelijk wordt.

Dit is ook het geval bij de Marindineesche verwantschapsnamen, die des te beter in de taal behouden bleven, wijl de familiebetrekkingen vaak niet door persoonsnamen (eigennamen) mogen worden vervangen; m. a. w. men noemt bloed- en aanverwanten meestal niet bij den eigennaam (soms is dit zelfs doerke of pemali), maar bij den familie-betrekkingsnaam, zóó, dat het soms lijkt alsof alle Marindineezen "familie» van elkaar zijn, wijl ze elkaar bijna altijd onder een of anderen familiebetrekkingsnaam aanspreken (zooals bij ons: vader, broer, oom, neef, etc.). Vandaar verder ook weer de vele grammaticale vormen dier namen, naargelang enkelvoud of meervoud, bezitsvorm van $1^{\mathrm{en}}, 2^{\mathrm{en}}$ en $3^{\mathrm{en}}$ persoon, vocatiefvorm, enz. Vele verwantschapsnamen immers hebben personalvormen om den bezitter aan te duiden, b.v. navai $=$ mijn vader; hava $=$ uw vader; eva 
$=$ zijn vader; ivind $=$ hun vaders, enz. Zoo heeft menige verwantschapsnaam drie bezitsvormen (naar gelang de bezitter $1^{\mathrm{e}}$, $2^{c}$ of $3^{c}$ persoon is), en dat wel in 't enkelvoud en meervoud, en bovendien nog een vocatiefvorm. De vormen voor de diverse verwantschapsnamen en familiebetrekkingen zijn dus zeer talrijk, en in den beginne bijna niet te ontwarren, - waarbij nog komt, dat de familierelaties véél nauwkeuriger en uitgebreider worden aangeduid dan bij ons.

Door onderstaande lijsten wordt geprobeerd zoo duidelijk mogelijk al die vormen weer te geven, - eerst wat de voornaamste spraakkunstige vormen betreft, - dan wat de ingewikkelde familierelaties aangaat. Voor een juiste uitspraak der Marindineesche woorden zij opgemerkt, dat de klemtoon op de laatste lettergreep valt.

\section{Grootvader :}

enk. $1^{\mathrm{e}}$ pers.: a mei(mijn, onze mrv. $1^{\mathrm{e}}$ pers.: nazè (mijn, onze grootvader) grootvaders)

» $2^{\mathrm{e}}$ pers.: h a z a m (uw gr.)

" $3^{\mathrm{e}}$ pers.: i $z$ a $\mathrm{m}(\mathrm{zijn}$, haar, hun gr.)

vocatief: amei.

» $2^{\mathrm{e}}$ pers.: haz c̀ (uw grootvaders)

$» \quad 3^{\mathrm{e}}$ pers.: izè (zijn, haar,hun gr.'s)

\section{Grootmoeder:}

enk. $1^{\mathrm{e}}$ pers.: $\mathrm{na}$ a o » $2^{e}$ pers.: hazoe » $3^{\text {e }}$ pers.: o ezoe mrv. $1^{\mathrm{e}}$ pers.: $\mathrm{n}$ aż̀ » $2^{\mathrm{e}}$ pers.: hazè , $3^{\mathrm{e}}$ pers.: iz è

vocatief: a mei.

Grootouders: iz è (ook: voorouders, voorvaderen).

Vader:

$$
\begin{aligned}
& \text { enk. } 1^{\mathrm{e}} \text { pers.: } \mathrm{navai} \\
& 2^{e} \text { pers.: havai } \\
& 3^{e} \text { pers.: evai } \\
& \text { mrv. } 1^{\mathrm{e}} \text { pers.: navind } \\
& \text { » } 2^{\mathrm{e}} \text { pers.: havind } \\
& » \quad 3^{e} \text { pers.: ivind }
\end{aligned}
$$

Moeder :

enk. $1^{\mathrm{e}}$ pers.: $\mathrm{n}$ avoe

》 $2^{\mathrm{e}}$ pers.: havoe

» $3^{\mathrm{e}}$ pers.: $w \bar{a} \mathrm{~h}$ mrv. $1^{\mathrm{e}}$ pers.: navi-sav

» $2^{e}$ pers.: havi-sav

$\gg 3^{\mathrm{e}}$ pers.: ivi-sav

vocatief; ån of $n \bar{e}$. - Ouders: ivind. 


\section{Echtgenoot:}

enk. $1^{\mathrm{e}}$ pers.: $\mathrm{nazam}$

» $2^{\mathrm{e}}$ pers.: hazam

- $3^{\mathrm{e}}$ pers.: ezam mrv. $1^{\mathrm{e}}$ pers.: nazoes

$\gg \quad 2^{\mathrm{e}}$ pers.: $\mathrm{h}$ azoes

$» 3^{e}$ pers.: izoes.

\section{Echtgenoote:}

vocatief: $\mathrm{nazam}$.

enk. $1^{\mathrm{e}}$ pers.: $\mathrm{nazoem}$

» $2^{\circ}$ pers.: hazoem

mrv. $1^{\mathrm{e}}$ pers.: $\mathrm{nazoes}$

» $3^{\text {e }}$ pers.: oezoem

» $2^{\mathrm{e}}$ pers.: hazoes

$\gg 3^{\mathrm{e}}$ pers.: izoes

vocatief: nazoem.

Zoon:

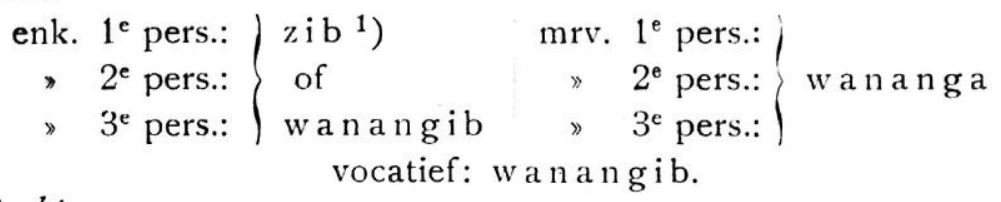
vocatief: wan angib.

\section{Dochter :}

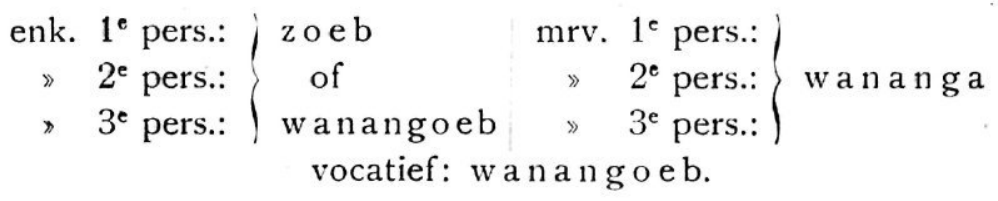

Kleinzoon en Kleindochter:

enk. $1^{\circ}$ pers.: $\mathrm{nazeb}$

» $2^{\circ}$ pers.: $\mathrm{hazeb}$

mrv. $1^{\mathrm{e}}$ pers.: $\mathrm{naz} \dot{\mathrm{e}}$

» $3^{\mathrm{e}}$ pers.: $\mathrm{izeb}$

» $2^{\mathrm{e}}$ pers.: hazè

" $3^{\mathrm{e}}$ pers.: iz è

vocatief: nazè, mrv. nazè.

Broeder :

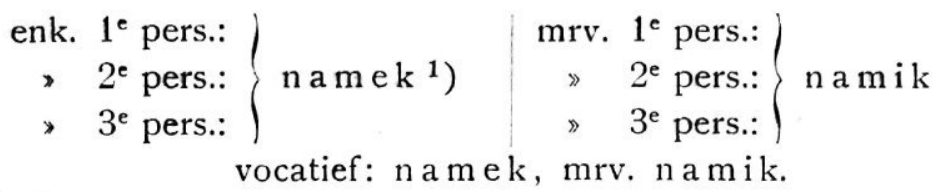

Zuster:

enk. $1^{\mathrm{e}}$ pers.:

» $2^{\text {e }}$ pers.: $\left\{\right.$ namoek ${ }^{1}$ ) $\gg 2^{\text {e }}$ pers.: namoek

" $3^{\mathrm{e}}$ pers.:, $3^{\mathrm{e}}$ pers.: !

vocatief: namoek, mrv. namik.

1) Wijl in deze woorden de possessief praefixen ontbreken, wordt de bezitsvorm uitgedrukt met behulp van het voorgevoegde bezittelijk voornaamwoord: nohan (mijn), wohan (uw), anep (zijn); nohan (onze), johan (uwe), anip (hun, haar). 
N.B. Oudste broer: mahai a $n$ e m

Middelste broer: in - an em Jongste broer: es-anem
Oudste zus: mahai-anoem

Middelste zus: in-anoem Jongste zus: es-anoem

Necf en Nicht:

enk. $1^{\mathrm{e}}$ pers.: onos

" $2^{\text {e }}$ pers.: hanos

mrv. $1^{\mathrm{e}}$ pers.: onos $^{2}$

$3^{e}$ pers.: izanos

$2^{\mathrm{e}}$ pers.: hanos ${ }^{2}$

$3^{\text {e }}$ pers.: izanos ${ }^{2}$

vocatief: on os.

N.B. Onos wordt alléén gebruikt in de beteekenis van: kind van moeders broer of zus, en kind van vaders zus. - Alle andere beteekenissen van neef en nicht worden ànders vertaald (zie verderop).

Oom :

enk. $1^{\mathrm{e}}$ pers.: $\mathrm{nahok}$

» 2 e pers.: hahok

\3 pers.: wahok mrv. $1^{\mathrm{e}}$ pers.: $\mathrm{naho \textrm {k } ^ { 2 }}$

$\gg 2^{\mathrm{e}}$ pers.: hahok ${ }^{2}$

» $3^{\mathrm{e}}$ pers.: wahok ${ }^{2}$

vocatief: bab.

Tante:

enk. $1^{\mathrm{e}}$ pers.: vavjak

- $2^{\mathrm{e}}$ pers.: havjak

$» 3^{\text {e }}$ pers.: evjak mrv. $1^{\mathrm{e}}$ pers.: bakāk

$\gg 2^{\text {e }}$ pers.: havjak ${ }^{2}$

» $3^{\mathrm{e}}$ pers.: evjak ${ }^{2}$

vocatief: $k \bar{a} \mathrm{k}$.

N.B. Nahok wordt alleen gebruikt in de beteekenis van: man van vaders zus, evenals voor ouderen broer van echtgenoot (zwager). Voor andere beteekenissen van "oom» zie verderop. - Zoo ook wordt vavjak alleen gebruikt voor tante in den zin van vaders zuster.

Schoonbroer (zwager):

I. Een man noemt den man van zijn jongere zuster: mand a (voc.)

enk. $1^{\mathrm{c}}$ pers.: $\mathrm{manda}$

» $2^{\mathrm{e}}$ pers.: $\mathrm{hamanda}$

\ $3^{\mathrm{e}}$ pers.: zimanda

II. Een vrouw noemt den man van haar jongere zuster; n a ba (voc.) mrv. $1^{\mathrm{e}}$ pers.: $\mathrm{manda}{ }^{2}$

» $2^{\mathrm{e}}$ pers.: $\mathrm{hamand \textrm {a } ^ { 2 }}$

» $3^{\mathrm{e}}$ pers.: $\mathrm{zimanda}{ }^{2}$ 
enk. $1^{\mathrm{e}}$ pers.: $\mathrm{naba}$

$» 2^{e}$ pers.: hanaba

$» \quad 3^{\mathrm{e}}$ pers.: $\mathrm{zinaba}$ mrv. 1' pers.: $\mathrm{naba}^{2}$

$\gg 2^{e}$ pers.: $\mathrm{hanaba}{ }^{2}$

\ $3^{e}$ pers.: zinaba ${ }^{2}$

III. Behuwd zwager, als de vrouwen gezusters zijn: nakom (voc.)

enk. $1^{\mathrm{e}}$ pers.: $\mathrm{nakom}$

» $2^{\mathrm{e}}$ pers.: hakom

» $3^{\mathrm{e}}$ pers.: zinakom mrv. $1^{\mathrm{e}}$ pers.: $\mathrm{nakom}{ }^{2}$

» $2^{\text {e }}$ pers.: hakom ${ }^{2}$.

- $3^{\text {e }}$ pers.: zinakom ${ }^{2}$

N.B. Voor de verschillende benamingen van schoonzuster (zwagerin) alsmede voor de nog andere beteekenissen van schoonbroer (zwager) zie verderop bij de familierelaties.

\section{Schoonvader:}

enk. $1^{\mathrm{e}}$ pers.: nakně $2^{e}$ pers.: hakne » $3^{e}$ pers.: ezakně vocatief: $p \bar{a} p$.

mrv. $1^{\mathrm{e}}$ pers.: nazakn è » $2^{e}$ pers.: hazakn \ $3^{e}$ pers.: ezakne

N.B. Dit heeft alleen de beteekenis van "schoonvader van een vrouw». - Voor "schoonvader van een man», alsmede voor de vertalingen van "schoonmoeder» zie verderop.

Schoonouders: (onderling)

enk. $1^{\mathrm{e}}$ pers.: n a pam

$\gg 2^{\mathrm{e}}$ pers.: hapam

$3^{\text {e }}$ pers.: iz pam

vocatief: pam.

mrv. $1^{\mathrm{e}}$ pers.: $\mathrm{napa \textrm {m } ^ { 2 }}$

$\rightarrow \quad 2^{\mathrm{e}}$ pers.: hapa $\mathrm{m}^{2}$

» $3^{\mathrm{e}}$ pers.: iz pa m ${ }^{2}$

Schoondochter:

enk. $1^{\mathrm{e}}$ pers.: nikne

$2^{e}$ pers.: hikne

$3^{\text {e }}$ pers.: izikn ̌

vocatief: nikn ̌.

N.B. "Schoonzoon» wordt vertaald door $\mathrm{nab}$ a, evenals "schoonbroer» (zie boven II).

Hiermede hebben we de voornaamste verbogen vormen van familiebetrekkingsnamen aangegeven. De vele onverbogen vormen vullen verder de lange lijst der diverse familiebetrekkingen aan, zoodat men bijna geen verwantschapsrelatie kan uit- 
denken, of men heeft er een apart woord voor, dat veelvuldig in den dagelijkschen omgang voorkomt. Men verbaast zich erg, hoe deze onontwikkelde menschen al die benamingen voor jongere en oudere, mannelijke en vrouwelijke bloed- en aanverwarten, zoo rap weten te gebruiken. Ik wil probeeren ze in een overzichtelijk lijstje te verzamelen, waarin dan de bovengenoemde verbogen vormen alleen in den bezitsvorm van den derden persoon zullen worden aangegeven, en voor de juiste vertaling vaak de Latijnsche benaming wordt toegevoegd. Vooraf zij nog opgemerkt, dat men als "kern» van de familiebetrekkingen neme het gezin: vader en moeder, zoon en dochter, broeder en zuster (evai, wāh; zib; zoeb; namek, namoek); waarbij men bedenke dat deze zes woorden vaak in zeer breeden zin worden genomen, b.v. namek is niet alleen broeder in de gewone beteekenis, maar ook: neef (kind van vaders broer), vriend, stamgenoot, totem-genoot, etc., en al de daaruit voortvloeiende verdere betrekkingen worden óók zoo opgevat, b.v. de vrouw van een "stamgenoot» (namek) heet ook "zwagerin» (naba) etc.

Voor de benamingen der verschillende relaties krijgen we dan het volgende. (Bezitsvormen der verbuigbare namen zie boven: hier wordt alleen vorm van $3^{\text {en }}$ pers. en vocatiefvorm aangegeven).

Man (vir):

e $\mathrm{zam}$, voc. $\mathrm{nazam}$

Vader (pater):

evai, voc. haz of hai Zoon (filius):

zib, voc. wanangib Broeder (frater):

$\mathrm{namek}$, voc. n a mek

$$
\left\{\begin{array}{c}
\text { oudere broeder: } \\
\text { mahai-anem } \\
\text { middelste broeder: } \\
\text { in-an e m } \\
\text { jongere broeder: } \\
\text { es-an em }
\end{array}\right.
$$

Grootvader (avus):

i z a $\mathrm{m}$, voc. a me i

Kleinzoon (nepos):

izeb, voc. nazeb
Vrouw (uxor):

o ezoem, voc. nazoem

Moeder (mater):

$\mathrm{w} \overline{\mathrm{a}} \mathrm{h}$, voc. $\stackrel{\mathrm{a}}{\mathrm{n}}$ of $\mathrm{n} \overline{\mathrm{e}}$

Dochter (filia):

zoeb, voc. wan a noeb

Zuster (soror):

n a moek, voc. namoek

oudere zuster:

mahai - anoem

middelste zuster:

in - a noem

jongere zuster:

es-anoem

Grootmoeder (avia):

o ezoe, voc. a me i

Kleindochter (neptis):

ize b, voc. nazeb 
Oom:

1. vaders broeder (patruus): evai, voc. haz vaders oudste broer: mahai-evai vaders middelste broer: $\left\{\begin{array}{c}\text { in - evai } \\ \text { vaders jongste broer: } \\ \text { es - evai }\end{array}\right.$

2. moeders broeder(avunculus): wahok, voc. bāb

3. man van vaders zuster (vir amitae): wa hok, voc. bāb

4. man van moeders zuster (vir materterae):

takev of evai, voc. hai

\section{Neef:}

1. zoon van vaders broer(frater patruelis):

namek, voc. namek $\left\{\begin{array}{l}\text { oudste: mahai-a nem } \\ \text { middelste: in - a n e m } \\ \text { jongste: es-an em }\end{array}\right.$

2. zoon van moeders broer (filius avunculi):

izanos, voc. onos

3. zoon van vaders zuster (amitinus):

izanos, voc. on os

4. zoon van moeders zuster

(filius materterae): iz anos, voc. on os

5. 'n man noemt den zoon van zijn broer (avunculinus): wan angib, voc. wanangib
Tante :

1. moeders zuster (matertera): $w \bar{a} h$, voc. ån

(moeders oudste zus: m a ha i-wāh moeders middelste zus: in - wa $\mathrm{a}$ moeders jongste zus: es - wa $\mathrm{h}$

2. vaders zuster (amita): evjak, voc. $k \bar{a} k$

3 . vrouw van moeders broer (avunculi uxor): wāh, voc. ån

4. vrouw van vaders ouderen broer (patrui sr. uxor): o ezoe, voc. a mei

5 . vrouw van vaders jongeren broer (patrui jr. uxor): $w \bar{a} h$, voc. ân

\section{Nicht:}

1. dochter van vaders broer (soror patruelis): n a moek, voc. namoek foudste: mahai-anoem middelste: in - a noem

! jongste: es - a noem

2. dochter van moeders broer (filia avunculi):

izanos, voc. onos

3. dochter van vaders zuster (amitina):

izanos, voc. onos

4. dochter van moeders zuster (filia materterae): izanos, voc. onos

5. 'n man noemt de dochter van zijn broer (avunculina): wanangoeb, voc. wanangoeb 
6. 'n man noemt den zoon van zijn zuster (consobrinus): oha-anem, voc. wanangib

7. 'n vrouw noemt den zoon van haarbroer(filius fratris): izibi, voc. kēmbra

8. 'n vrouw noemt den zoon van haar zuster (sororius): wan angib, voc. wanangib

9. oudere neef van echtgenoot(e) (nepos sr.):

mahai-rik, voc. namek

10. jongere neef van echtgenoot(e) (nepos jr.): es-rik, voc. namek

\section{Schoonvader:}

1. 'n vrouw noemt den vader van haar man (socer major): ezakně, voc. pāp

2 . 'n man noemt den vader van zijn vrouw (socer minor): $\mathrm{z}$ in a ba, voc. $\mathrm{naba}$

N.B. Schoonouders van den man: $z$ in a b a ${ }^{2}$

Schoonouders van devrouw: es a n è

Schoonouders onderling: iz pam, voc. pam

\section{Schoonzoon :}

dochters man (gener): $\mathrm{z}$ in $\mathrm{aba}$, voc. $\mathrm{naba}$

Schoonbroer (zwager):

1. 'n vrouw zegt tot ouderen broer van haar man (mansbroer: levir sr.): wahok, voc. nahok
6. 'n man noemt de dochter van zijn zuster(consobrina): oha-anoem, voc. wanangoeb

7. 'n vrouw noemt de dochter van haar broer (filia fratris): izibi, voe. $k \bar{e} m b r a$

8. 'n vrouw noemt de dochter van haar zuster (sororia): wa nangoeb, voc. wanangoeb

9. oudere nicht van echtgenoot(e) (neptis sr.):

mahai-roek, voc. namoek

10. jongere nicht van echtgenoot(e) (neptis jr.):

es-roek, voc. namoek

\section{Schoonmoeder:}

1. 'n vrouw zegt tot de moeder van haar man (socrus major): evjak, voc. $k \bar{a} k$

2. 'n man zegt tot de moeder van zijn vrouw (socrus minor): zinaba, voc. naba.

\section{Schoondochter:}

zoons vrouw (nurus):

iziknĕ, voc. nikn

Schoonzuster (zwagerin):

1. 'n vrouw zegt tot de oudere zuster van haar man (manszuster: glos sr.):

esanè, voc. mahai-roek 
2. 'n vrouw zegt tot jongeren broer van haar man (mansbroer: levir jr.):

wan angib, voc. wa nangib

3. 'n manzegt tot ouderen broeder van zijn vrouw (vrouwsbroer: uxoris frater sr.): zim a nda, voc. manda

4. 'n man zegt tot jongeren broeder van zijn vrouw (vrouwsbroer: uxoris frater jr.): savok, voc. wanangib

5. 'n man zegt tot den man van zijn oudere zuster (zustersman: vir sororis sr.): zambit-evai, voc. mbit

6. 'n man zegt tot den man van zijn jongere zuster (zustersman: vir sororis jr.):

$z$ imanda, voc. manda

7. 'n vrouw zegt tot den man van haar of 's mans oudere zuster (vir sororius sr.):

nasoe, voc. mahai-roek

8 . 'n vrouw zegt tot den man van haar of 's mans jongere zuster (vir sororius jr.): $z$ in aba, voc. naba

9. behuwd zwager (als de vrouwen gezusters zijn : levir collateralis):

zinakom, voc. nakom
2. 'n vrouw zegt tot de jongere zuster van haar man (manszuster: glos jr.):

nakaroe, voc. es-roek

3.'n man zegt tot de oudere zuster van zijn vrouw (vrouwszuster: uxoris soror sr.): $z$ in aba, voc. naba

4. 'n man zegt tot jongere zuster van zijn vrouw (vrouwszuster: uxoris soris jr.): savok, voc. wan angoeb

5. 'n man zegt tot de vrouw van zijn ouderen broer (broersvrouw: uxor fratris sr.): $w \bar{a} h$, voc. ån

6. 'n man zegt tot de vrouw van zijn jongeren broer (broersvrouw: uxor fratris jr.): esanè, voc. es-roek

7. 'n vrouw zegt tot de vrouw van haar of 's mans ouderen broer (uxor fratris sr.): $w \bar{a} h$, voc. ån

8. 'n vrouw zegt tot de vrouw van haar of 's mans jongeren broer (uxor fratiria jr.): wāh, voc. ån

9. behuwd zwagerin (als de mannen gebroeders zijn (glos collateralis): zin a kom, voc. nakom

.B. Om deze ingewikkelde benamingen voor $\mathrm{schoonbroer}$ en schoonzuster wat te vergemakkelijken, kan het volgende voorbeeld dienen: Stel dat Jan getrouwd is met Cor, en dat we hebben:

Jan's oudere broèr Piet is getrouwd met Bertha jongere broer Frans

Cato

* oudere zuster Anna

» jongere zuster Maria

- Hendrik Frits 
Cor's oudere broer Antoon is getrouwd met Rica

, jongere broer Theo " , Dora

》 oudere zuster Lisa, " Bertus

» jongere zuster Teresia » "Jacobus

Schoonbroer is dan: 1. Piet ten opzichte van Cor. 2. Frans t. n. v. Cor. 3. Antoon t. o. v. Jan. 4. Theo t. o. v. Jan. 5. Hendrik t. o. v. Jan. 6. Frits t. o. v. Jan. 7. Bertus en Hendrik t. o. v. Cor. 8. Jacobus en Frits t. o. v. Cor. 9. Hendrik t. o. v. Frits en Bertus t. o. v. Jacobus.

Schoonzuster is dan: 1. Anna t. o. v. Cor. 2. Maria t. o. v. Cor. 3. Lisa t. o. v. Jan. 4. Teresia t. o. v. Jan. 5. Bertha t. o. v. Jan. 6. Cato t. o. v. Jan. 7. Bertha en Rica t. o. v. Cor. 8. Cato en Dora t. o. v. Cor. 9. Bertha t. o.v. Cato en Rica t. o. v. Dora.

Stiefrader (vitricus):

kisih-evai, voc. haz

Stiefzoon (privignus):

kis ih - wa na ngib, voc. wanangib

Halfbroer (frater conjugalis): kisih-namek, voc. namek

Pleegvader (pater adoptans): ${ }^{1}$ )

1. van een jongen:

binahor-evai of ketakob-evai, voc. haz

2. van een meisje:

jarang-evai, voc. haz.

N.B. Pleegouders van jongen:

binahor-ivind

Pleegouders van meisje: jarang-ivind

Pleegzoon (filius adoptivus):

binahor, voc. wanangib
Stiefmoeder (noverca): kisih-wāh, voc. $n \bar{e}$

Stiefdochter (privigna): kisih-wanangoeb, voc. wanangoeb

Halfzuster (soror conjugalis): kisih-namoek, voc.namoek

Pleegmoeder (mater adoptans):

1. van een jongen:

binahor-wāh of ketakob-wāh, voc. ne

2. van een meisje: jarang-wāh, voc. $n \bar{e}$

Pleegdochter (filia adoptiva): jarang, voc. wanangoeb

1) Het aannemen van andermans kinderen is algemeen bij de Marindineezen, en behoort bij het ethnologisch verschijnsel dat men "fosterage" heeft genoemd. 
Men zou hier verder voor de volledigheid nog enkele benamingen bij kunnen voegen, als:

Eerste man: mahai-ezam

Tweede man: es-ezam

Verloofde (jongen): so r a m

Weduwnaar: boi-rik

Kindje (jongen): papis

Jongen: patoer

Jongeling: e wat i

Huwbaar jongeling: $\mathrm{miak}$ i m

Man: a mnangib

Ongetrouwd man: moharik

Jonggehuwd man: noh-anem

Oude man: somb-anem
Eerste, vrouw: mahai-oezoem Tweede vrouw: es-oezoem Verloofde (meisje): soram Weduwe: boi-roek

Kindje (meisje): papoes

Meisje: kiva som

Jonge meid: wahoekoe Huwbaar meisje: iwag

Vrouw: sav

Ongetrouwde vrouw: mo ha roek Jonggehuwde vrouw: noha noem

Oude vrouw: mes-iwag

Voor «kind" (in 't algemeen) heeft men: hon-a-hon (wichtje), narakam, seis (kleintje), nin (gestolen kind), is ahis (de kleinen), etc. - Voor "makker» (ml. en vr.) heeft men izatom, waarvan de vocatief is ngeis. - Getrouwde lui heeten met een algemeenen naam: hizoes-ti. - De mannen: has-ti; de vrouwen: boeb-ti, nova-ti, awin-ti, etc.

Men ziet uit dit alles dat de familiebetrekkingen en andere relaties bij de Marindineezen zeer uitvoerig worden benoemd. Bizondere vermelding verdienen nog de vocatief-vormen mbit, manda, naba, nakom, pāp, nikně, pam, die "verplichtend» zijn voor de desbetreffende bloed- en aanverwanten: hun eigennaam noemen is doerk e (pemali).

Het kost heel wat inspanning om in de terminologie der Marindineesche maatschappij thuis te raken, en het zou wel interessant kunnen zijn om in verband daarmee het heerschend patriarchaat, de exogamie en het totemisme, die met al die benamingen nauw samenhangen, te bestudeeren; doch het geduld van de lezers is al genoeg op de proef gesteld! 